\title{
Diet-obesity associations in children: approaches to counteract attenuation caused by misreporting
}

Claudia Börnhorst ${ }^{1}$, Inge Huybrechts ${ }^{2,3}$, Antje Hebestreit ${ }^{1}$, Barbara Vanaelst ${ }^{2,4}$, Dénes Molnár ${ }^{5}$, Silvia Bel-Serrat ${ }^{6}$, Theodora Mouratidou ${ }^{6}$, Luis A Moreno ${ }^{6}$, Valeria Pala ${ }^{7}$, Marge Eha ${ }^{8}$, Yiannis A Kourides 9 , Alfonso Siani ${ }^{10}$, Gabriele Eiben ${ }^{11}$ and Iris Pigeot ${ }^{1, *}$, on behalf of the IDEFICS consortium

${ }^{1}$ BIPS - Institute for Epidemiology and Prevention Research, Achterstrasse 30, 28359 Bremen, Germany:

${ }^{2}$ Department of Public Health, Ghent University, Ghent, Belgium: ${ }^{3}$ International Agency for Research on Cancer

(IARC), Dietary Exposure Assessment Group (DEX), Lyon, France: ${ }^{4}$ Research Foundation-Flanders (FWO),

Brussels, Belgium: ${ }^{5}$ Medical Faculty, Department of Pediatrics, University of Pécs, Pécs, Hungary: ${ }^{6}$ GENUD

(Growth, Exercise, Nutrition and Development) Research Group, University of Zaragoza, Zaragoza, Spain:

${ }^{7}$ Fondazione IRCSS, Istituto Nazionale dei Tumori, Department of Preventive and Predictive Medicine, Nutritional Epidemiology Unit, Milan, Italy: ${ }^{8}$ Department of Surveillance and Evaluation, National Institute for Health Development, Tallinn, Estonia: ${ }^{9}$ Research and Education Foundation of Child Health, Paphos, Cyprus:

${ }^{10}$ Institute of Food Sciences, CNR, Avellino, Italy: ${ }^{11}$ Department of Public Health and Community Medicine, University of Gothenburg, Gothenburg, Sweden

Submitted 23 February 2012: Final revision received 8 August 2012: Accepted 24 August 2012: First published online 90 October 2012

\begin{abstract}
Objective: Measurement errors in dietary data lead to attenuated estimates of associations between dietary exposures and health outcomes. The present study aimed to compare and evaluate different approaches of handling implausible reports by exemplary analysis of the association between dietary intakes (total energy, soft drinks, fruits/vegetables) and overweight/obesity in children.

Design: Cross-sectional multicentre study.

Setting: Kindergartens/schools from eight European countries participating in the IDEFICS Study.

Subjects: Children ( $n$ 5357) aged $2-9$ years who provided one $24 \mathrm{~h}$ dietary recall and complete covariate information.

Results: The $24 \mathrm{~h}$ recalls were classified into three reporting groups according to adapted Goldberg cut-offs: under-report, plausible report or over-report. In the basic logistic multilevel model (adjusted for age and sex, including study centre as random effect), the dietary exposures showed no significant association with overweight/obesity (energy intake: OR=0.996 (95\% CI 0.983, 1.010); soft drinks: OR $=0.999(95 \%$ CI $0.986,1.013))$ and revealed even a positive association for fruits/vegetables (OR $=1.009$ (95\% CI 1.001, 1.018)). When adding the reporting group (dummy variables) and a propensity score for misreporting as adjustment terms, associations became significant for energy intake as well as soft drinks (energy: OR $=1.074$ (95\% CI 1.053, 1.096); soft drinks: OR $=1.015(95 \%$ CI $1 \cdot 000,1 \cdot 031)$ ) and the association between fruits/vegetables and overweight/obesity pointed to the reverse direction compared with the basic model (OR $=0.993$ (95\% CI 0.984, 1.002)).

Conclusions: Associations between dietary exposures and health outcomes are strongly affected or even masked by measurement errors. In the present analysis consideration of the reporting group and inclusion of a propensity score for misreporting turned out to be useful tools to counteract attenuation of effect estimates.
\end{abstract}

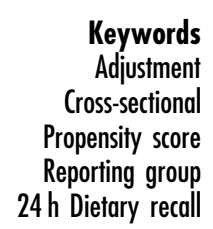

Measurement errors in dietary variables pose a challenge for epidemiologists when investigating associations between dietary intakes and health outcomes ${ }^{(1)}$. Problems in particular emerge from misreporting, which comprises under-reporting and over-reporting. Several studies have revealed that misreporting is characteristic to specific individuals and results in differential errors ${ }^{(2-4)}$. Differential errors are related to the outcome of interest and induce bias such that associations between dietary factors and health outcomes may be attenuated, exaggerated or hidden ${ }^{(5)}$, 
whereas non-differential (random) errors tend to attenuate associations. Various procedures have been proposed to screen out implausible dietary recalls ${ }^{(6,7)}$ but the question how to handle recalls identified as implausible is still open.

Researchers commonly refer to validation studies that confirm the accuracy/reliability of their assessment instruments but do not consider misreporting in the later analyses, although there are different procedures that could be applied $^{(8,9)}$ : (i) exclusion of inaccurate recalls; (ii) adjustment for the reporting group (under-report, plausible report, over-report); (iii) stratified analysis by reporting group; and (iv) propensity score adjustment.

Despite several studies having found that exclusion of under-reports strengthened diet-obesity relationships ${ }^{(3,10,11)}$, data exclusions may introduce a source of unknown bias and has not been recommended ${ }^{(12)}$. Adjusting for the reporting group seems an appropriate alternative to data exclusions and was shown to yield consistent results compared with those obtained from plausible reports in stratified analyses $^{(10)}$. Although not applied in this context yet, the propensity score is a common tool to reduce bias by equating groups based on selected covariables. A propensity score reflects the conditional probability of assignment to a particular group given a vector of observed covariables ${ }^{(13)}$. Construction of a propensity score based on variables previously found to be related to misreporting could be another option to account for implausible recalls.

Studies in adults investigating the handling of implausible recalls are rare ${ }^{(8,9,14)}$. To the authors' knowledge, no study to date has addressed this issue in children. As dietary recalls in young children often rely on proxy reports ${ }^{(15)}$, it is likely that misreporting is triggered by different factors compared with adults (e.g. unintentional under-reporting due to lack of parental control). The present study aimed to evaluate the four different approaches to account for misreporting in the statistical analysis mentioned above and finally to give recommendations on how to handle the problem of inaccurate reports in future studies on dietary behaviour in children.

\section{Materials and methods}

\section{Study population}

IDEFICS (Identification and prevention of Dietary- and lifestyle-induced health EFfects In Children and infantS) is a multicentre, setting-based study aiming to prevent and investigate the causes of diet- and lifestyle-related diseases like overweight and obesity in European children aged 2-9 years. The baseline survey was conducted from September 2007 to June 2008; more than 31500 children were contacted, out of whom finally 16220 participated and fulfilled the inclusion criteria of the IDEFICS Study. Children were recruited through kindergartens/schools. In addition to self-completion questionnaires, interviews with parents concerning lifestyle habits and dietary intakes as well as anthropometric measurements and examinations of the children were conducted in examination centres, which were the settings in most countries. All measurements were taken by trained study personnel using standardised procedures in all eight study centres (Belgium, Cyprus, Estonia, Germany, Hungary, Italy, Spain and Sweden). Details on the design and objectives of the study are given elsewhere $^{(16,17)}$.

\section{Etbics approval}

Applicable institutional and governmental regulations regarding the ethical use of human volunteers were followed during this research. Approval of the appropriate ethics committees was obtained by each of the eight participating centres carrying out the fieldwork (Belgium: Ethics Committee, University Hospital, Ghent; Cyprus: Cyprus National Bioethics Committee; Estonia: Tallinn Medical Research Ethics Committee; Germany: Ethics Committee, Universtiy of Bremen; Hungary: Egészségügyi Tudományos Tanács, Pécs; Italy: Comitato Etico, Avellino; Spain: Comité Ético de Investigación, Clínica de Aragón (CEICA); Sweden: Regional Ethics Review Board, University of Gothenburg).

Parents provided written informed consent for all examinations. Each child was informed orally about the modules by field workers and asked for his/her consent immediately before examination ${ }^{(17)}$. Study children did not undergo any procedure before both they and their parents gave consent for examinations, collection of samples, subsequent analysis and storage of personal data and collected samples. Participants and their parents could consent to single components of the study while abstaining from others.

\section{Anthropometry}

Height (centimetres) of the children was measured to the nearest $0 \cdot 1 \mathrm{~cm}$ with a calibrated statiometer (Seca 225; Seca, Birmingham, UK); body weight (kilograms) was measured in light underwear on a calibrated scale accurate to $0 \cdot 1 \mathrm{~kg}$ (Tanita BC 420 SMA; Tanita Europe GmbH, Sindelfingen, Germany). BMI was calculated as weight divided by height squared and the children were categorised according to the International Obesity Taskforce criteria $^{(18,19)}$. According to these criteria, centile curves corresponding to a BMI of $25 \mathrm{~kg} / \mathrm{m}^{2}$ and $30 \mathrm{~kg} / \mathrm{m}^{2}$ at age 18 years are chosen as extrapolation into childhood of the well-accepted adult cut-offs to define overweight/obesity, respectively. Thin and normal-weight children, as well as overweight and obese children, were combined into one category each to construct a binary outcome measure to be included in the logistic model.

\section{Dietary data}

Dietary data were assessed using the computerised $24 \mathrm{~h}$ dietary recall (24-HDR) SACINA (Self-Administered Children and Infants Nutrition Assessment), which is 
based on the previously designed and validated HELENADIAT $^{(20)}$ instrument that was originally developed for Flemish adolescents ${ }^{(21)}$. SACINA is structured according to six meal occasions (breakfast, morning snack, lunch, afternoon snack, dinner, evening snack) related to a range of chronological daily activities. For each food item the participant selects the consumed quantity by means of pictures with increasing portion sizes (based on predefined standard amounts) that are displayed on the screen to facilitate estimation of portion sizes. The intake of the food item is calculated then as the product of the reported quantity and the standard amount (e.g. 4 spoons of sauce at $15 \mathrm{~g}=60 \mathrm{~g}$ ). Proxies, mainly the parents, completed the 24-HDR under supervision of field personnel which lasted 20-30 min. In case the child had lunch at school on weekdays, school meals were additionally assessed by means of direct observation. Trained observers, teachers or caregivers entered portion sizes of all consumed foods and drinks on predefined assessment sheets. The uniquely coded food items were linked to country-specific food composition tables. Missing quantities for single food items as well as obviously implausible data entries were imputed by country-, food group- and age-specific median intakes $(0 \cdot 01 \%$ of the entries) to avoid excessive recall exclusions. Incomplete interviews were excluded, e.g. if the proxy did not know about at least one main meal or in the case of missing school meal information ( $n$ 2518). Furthermore, intakes of energy $>16736 \mathrm{~kJ} / \mathrm{d} \quad(>4000 \mathrm{kcal} / \mathrm{d})$ which seemed to be a result of computer or data-entry errors rather than of misreporting (e.g. several repeated entries for the same food item) were excluded ( $n$ 10). Although up to six repeated 24-HDR were carried out in a smaller sample, only the first recall day was included in the current analysis (including weekdays and weekend days) to obtain an equal number of 24-HDR for each child. The assessment procedure was slightly different in the Hungarian study centre, where dietary recalls were not performed via the standardised SACINA software but via paper-and-pencil 24-HDR registrations that were entered in the SACINA software afterwards. As this increased data heterogeneity and further seemed to affect the misreporting behaviour, data from Hungary were not considered in the present analyses. A study sample based on equal procedures and standardised assessment instruments was needed for this exploratory methodological study.

Energy intake (EI; kJ/d), fruit/vegetable intake and soft drink intake (as a percentage of total daily EI; \%EI) were used as exposure measures in the different models as these were repeatedly proposed to be associated with overweight/obesity ${ }^{(22-24)}$.

\section{Statistical methods}

Classification of $24 \mathrm{~h}$ dietary recalls

The BMR was estimated from the equations published by Schofield ${ }^{(25)}$ and recommended by the FAO/WHO/United
Nations University (1985) taking into account age, sex, body height and weight. To determine whether reported EI was consistent with energy requirements, the ratio of proxy-reported EI to predicted BMR was used to classify the 24-HDR into under-reports (UdR), plausible reports (PR) and over-reports (OvR) according to Goldberg et $a l^{(6)}$. Since the original Goldberg cut-offs were developed for adults and do not consider differences in EI due to age and sex, cut-off values were re-calculated for application in children as suggested previously ${ }^{(2,26)}$ using the formula:

$$
\text { Cut-off }=\mathrm{PAL} \times \exp \left[ \pm 1.96 \times \frac{(S / 100)}{\sqrt{n}}\right]
$$

where

$$
S=\sqrt{\frac{\mathrm{CV}_{\mathrm{wEI}}^{2}+\mathrm{CV}_{\mathrm{wBMR}}^{2}+\mathrm{CV}_{\mathrm{PA}}^{2}}{d}}
$$

The within-subject $\mathrm{CV}$ for $\mathrm{EI}\left(\mathrm{CV}_{\mathrm{wEI}}\right)$, the within-subject $\mathrm{CV}$ for BMR $\left(\mathrm{CV}_{\mathrm{wBMR}}\right)$ and the $\mathrm{CV}$ for physical activity $\left(\mathrm{CV}_{\mathrm{PA}}\right)$ were replaced by age- and sex-specific values as given in Nelson et $a l^{(27)}$ and Black et $a l^{(28)}$. Goldberg's overall physical activity level (PAL) of 1.55 was substituted by age- and sex-dependent levels of light physical activity (2-5 years: $1 \cdot 45$; $6-10$ years: males $1 \cdot 55$, females $1 \cdot 50)$ according to Torun et $a l^{(29)}$. The number of days (d) was set to 1 (one 24-HDR per child) to account for the large day-to-day variation in diet. Cut-off limits need to be wider if only one or few recall days are available as these may not reflect usual intakes but exceptional days. The resulting age- and sex-specific cut-off values to define UdR, PR and OvR are given in Table 1, which were then used to classify the recalls accordingly.

\section{Calculation of the propensity score}

In a previous study based on the IDEFICS data ${ }^{(30)}$, backward elimination in the course of multilevel logistic regression analysis was applied to identify factors significantly related to misreporting in proxy reports for young children. The covariables that turned out to be significantly associated with misreporting were used in the construction of the propensity score: age and sex of the child ${ }^{(31,32)}$, net household income (dummy: high $v$. medium/low), number of persons below 18 years of age in the household and day

Table 1 Lower and upper cut-off limits to classify $1 \mathrm{~d} 24-\mathrm{HDR}$ as UdR or OvR based on El:BMR

\begin{tabular}{lccc}
\hline Age (years) & Sex & $\begin{array}{c}\text { Lower cut-off } \\
(\text { UdR) }\end{array}$ & $\begin{array}{c}\text { Upper cut-off } \\
\text { (OvR) }\end{array}$ \\
\hline $2-<6$ & Boys & 0.74 & $2 \cdot 85$ \\
$2-<6$ & Girls & 0.78 & 2.69 \\
$6-<10$ & Boys & 0.92 & 2.61 \\
$6-<10$ & Girls & 0.93 & 2.43 \\
\hline
\end{tabular}

24-HDR, $24 \mathrm{~h}$ dietary recall; UdR, under-report; OvR, over-report; El, energy intake.

PR (plausible report) has El:BMR within the cut-offs. 
of the interview (dummy: weekday $v$. Saturday/Sunday). The following information on parental concerns and perception of their child's weight status obtained from a self-administered proxy questionnaire was included: 'How concerned are you about your child... (i) becoming overweight?; (ii) becoming underweight?' (response categories were 'unconcerned', 'a little concerned', 'concerned' and 'very concerned'); 'Do you think your child is... (i) 'much too underweight?'; (ii) 'slightly too underweight?'; (iii) 'proper weight?'; (iv) 'slightly too overweight'; (v) 'much too overweight?' (response categories were 'yes' and 'no'). Further intakes from the following food items commonly perceived to be healthy/unhealthy were considered as predictors for misreporting: chocolate products, other sugary products (e.g. cakes, biscuits, ice cream), soft drinks, fruits/vegetables, milk (all as \%EI) and water $(\mathrm{g} / \mathrm{d})$. Although BMI is a repeatedly shown predictor of misreporting, it was not included in the construction of the propensity score as the weight status is the outcome variable in the present analysis.

The conditional probability (propensity score) of being classified as UdR given the mentioned covariables was calculated applying a logistic multilevel regression model including all covariates mentioned above as fixed effects and the study centre as random effect:

\section{Propensity score $=$ estimated $P(\mathrm{UdR} \mid$ covariates $)$.}

Fruit/vegetable intake was not included as a covariable in the propensity score calculation when investigating dietobesity models using fruit/vegetable intake as exposure variable. Analogously, soft drink intake was not considered in the construction of the propensity score when investigating models using soft drink intake as exposure.

\section{Model building}

Associations between overweight/obesity and dietary intakes were exemplarily analysed to investigate different procedures of handling implausible dietary recalls. Logistic multilevel regression analyses were conducted using a dummy indicating overweight/obesity as outcome and the three dietary variables as exposure measures: EI in $\mathrm{kJ} / \mathrm{d}$ (models labelled with 'a'), \%EI from fruits/ vegetables (labelled with ' $b$ ') and \%EI from soft drinks (labelled with 'c').

The first model (basic model) included only adjustment terms for age and sex and a random effect for the study centre to account for the clustered study design (Model $1 \mathrm{a}-\mathrm{c})$. The basic model was also run adding all variables used in the calculation of the propensity score as potential confounders (Model $2 \mathrm{a}-\mathrm{c}$ ). Model 3 was identical to the basic model but here recalls classified as UdR and OvR were excluded. Further, the basic model was run adjusting additionally for the reporting group (Model $4 \mathrm{a}-\mathrm{c}$ ), for the propensity score (Model $5 \mathrm{a}-\mathrm{c}$ ) or for both (Model $6 \mathrm{a}-\mathrm{c}$ ). In addition, the basic model was analysed stratified by reporting group (Model $7 \mathrm{a}-\mathrm{c}$ ) as well as stratified by reporting group and at the same time adjusted for the propensity score (Model $8 \mathrm{a}-\mathrm{c}$ ).

The current analysis includes only children with $24-H D R$ and complete covariate information ( $n$ 5962). All analyses were performed using the statistical software package SAS version $9 \cdot 1$.

\section{Results}

Descriptive analyses of the study population and all covariables used for the construction of the propensity score are presented in Table 2 (categorical variables) and Table 3 (continuous variables). Regarding the total study group, $6 \cdot 7 \%(n 402)$ of the proxy reports were classified as UdR and $4.0 \%$ ( $n$ 241) as OvR. Both UdR and OvR were slightly higher in girls compared with boys and higher in the low/medium compared with the high income group. Percentages of UdR were higher in overweight/obese children, in the older age group ( 6 to $<10$ years), on weekend days and if proxies were concerned about their child becoming overweight or perceived their child to be slightly/much too overweight. OvR, on the other hand, was higher in thin/normal-weight children, on weekend days or if proxies were concerned about their child becoming underweight. \%EI from fruits/vegetables was highest in UdR whereas \%EI from chocolate and other sugary products were highest in OvR. Soft drink consumption was slightly lower in the OvR group compared with the UdR and PR groups.

Tables 4 and 5 show the odds ratios and 95\% confidence intervals obtained from the different models for the association between overweight/obesity and the three dietary exposures. Effects of continuous variables are assessed as 1-unit offsets from the mean; e.g. the OR for the association between overweight/obesity and \%EI from fruits/vegetables indicates the increase in risk when increasing \%EI from fruits/vegetables by $1 \%$ compared with the mean of the total study population.

In the basic model (Table 4, Models 1a-c), odds ratios were not significant for EI and soft drink intake and indicated even a significant positive association between overweight/obesity and fruit/vegetable intake $(\mathrm{OR}=1 \cdot 009$, 95\% CI 1·001, 1·018). Adjustment for covariables (Models $2 \mathrm{a}-\mathrm{c})$ revealed similar results, but the association between fruits/vegetables and overweight/obesity was rendered insignificant here (OR $=1.009$ (95\% CI 0.998, 1.020)). When excluding UdR and OvR (Models $3 \mathrm{a}-\mathrm{c}$ ), a significantly positive association between EI and overweight/ obesity was observed (OR $=1 \cdot 057,95 \%$ CI 1.038, 1.076). Adjustment for the reporting group (Models $4 \mathrm{a}-\mathrm{c}$ ) also revealed a significantly positive association between EI and overweight/obesity that was even slightly more pronounced compared with the model excluding misreports. When adjusting for the propensity score, all associations were strengthened (Models $5 \mathrm{a}-\mathrm{c}$ ) with the association 
Table 2 Descriptive analyses of categorical covariables stratified by reporting group (total numbers and row percentages): children aged 2-9 years, IDEFICS Study

\begin{tabular}{|c|c|c|c|c|c|c|c|}
\hline & \multirow{2}{*}{$\frac{\text { Total }}{n}$} & \multicolumn{2}{|c|}{ UdR } & \multicolumn{2}{|c|}{ PR } & \multicolumn{2}{|c|}{ OvR } \\
\hline & & $n$ & $\%$ & $n$ & $\%$ & $n$ & $\%$ \\
\hline All & 5962 & 402 & $6 \cdot 7$ & 5319 & $89 \cdot 2$ & 241 & $4 \cdot 0$ \\
\hline \multicolumn{8}{|l|}{ Sex of the child } \\
\hline Male & 3029 & 187 & $6 \cdot 2$ & 2747 & $90 \cdot 7$ & 95 & $3 \cdot 1$ \\
\hline Female & 2933 & 215 & $7 \cdot 3$ & 2572 & $87 \cdot 7$ & 146 & $5 \cdot 0$ \\
\hline \multicolumn{8}{|l|}{ Age groups } \\
\hline $2-<6$ years & 2625 & 120 & $4 \cdot 6$ & 2388 & $91 \cdot 0$ & 117 & $4 \cdot 5$ \\
\hline $6-<10$ years & 3337 & 282 & $8 \cdot 5$ & 2931 & $87 \cdot 8$ & 124 & $3 \cdot 7$ \\
\hline \multicolumn{8}{|l|}{ Weight status* } \\
\hline Thin/normal weight & 4721 & 249 & $5 \cdot 3$ & 4263 & $90 \cdot 3$ & 209 & $4 \cdot 4$ \\
\hline Overweight/obese & 1241 & 153 & $12 \cdot 3$ & 1056 & $85 \cdot 1$ & 32 & $2 \cdot 6$ \\
\hline \multicolumn{8}{|l|}{ Study centre } \\
\hline Belgium & 310 & 29 & $9 \cdot 4$ & 274 & $88 \cdot 4$ & 7 & $2 \cdot 3$ \\
\hline Cyprus & 403 & 63 & $15 \cdot 6$ & 335 & $83 \cdot 1$ & 5 & $1 \cdot 2$ \\
\hline Estonia & 602 & 35 & $5 \cdot 8$ & 537 & $89 \cdot 2$ & 30 & $5 \cdot 0$ \\
\hline Germany & 1504 & 159 & $10 \cdot 6$ & 1290 & $85 \cdot \overline{8}$ & 55 & $3 \cdot 7$ \\
\hline Italy & 1492 & 68 & $4 \cdot 6$ & 1320 & $88 \cdot 5$ & 104 & $7 \cdot 0$ \\
\hline Spain & 525 & 7 & $1 \cdot 3$ & 492 & $93 \cdot 7$ & 26 & $5 \cdot 0$ \\
\hline Sweden & 1126 & 41 & $3 \cdot 6$ & 1071 & $95 \cdot 1$ & 14 & $1 \cdot 2$ \\
\hline \multicolumn{8}{|l|}{ Income } \\
\hline Low/medium & 4304 & 322 & $7 \cdot 5$ & 3786 & $88 \cdot 0$ & 196 & $4 \cdot 6$ \\
\hline High & 1658 & 80 & $4 \cdot 8$ & 1533 & $92 \cdot 5$ & 45 & $2 \cdot 7$ \\
\hline \multicolumn{8}{|l|}{ Day of the interview } \\
\hline Weekday & 4925 & 319 & $6 \cdot 5$ & 4415 & $89 \cdot 6$ & 191 & 3.9 \\
\hline Saturday/Sunday & 1037 & 83 & $8 \cdot 0$ & 904 & $87 \cdot 2$ & 50 & $4 \cdot 8$ \\
\hline \multicolumn{8}{|c|}{ Concerned: child becoming underweight } \\
\hline Unconcerned & 3109 & 230 & $7 \cdot 4$ & 2796 & $89 \cdot 9$ & 83 & $2 \cdot 7$ \\
\hline A little concerned & 923 & 57 & $6 \cdot 2$ & 825 & $89 \cdot 4$ & 41 & $4 \cdot 4$ \\
\hline Concerned & 863 & 52 & $6 \cdot 0$ & 751 & $87 \cdot 0$ & 60 & $7 \cdot 0$ \\
\hline Very concerned & 1067 & 63 & $5 \cdot 9$ & 947 & $88 \cdot 8$ & 57 & $5 \cdot 3$ \\
\hline \multicolumn{8}{|c|}{ Concerned: child becoming overweight } \\
\hline Unconcerned & 3299 & 182 & $5 \cdot 5$ & 2996 & $90 \cdot 8$ & 121 & $3 \cdot 7$ \\
\hline A little concerned & 1001 & 73 & $7 \cdot 3$ & 879 & $87 \cdot 8$ & 49 & 4.9 \\
\hline Concerned & 878 & 70 & $8 \cdot 0$ & 774 & $88 \cdot 2$ & 34 & 3.9 \\
\hline Very concerned & 784 & 77 & $9 \cdot 8$ & 670 & $85 \cdot \overline{5}$ & 37 & $4 \cdot 7$ \\
\hline \multicolumn{8}{|l|}{ Health: child's weight } \\
\hline Much too underweight & 77 & 6 & $7 \cdot 8$ & 66 & $85 \cdot 7$ & 5 & $6 \cdot 5$ \\
\hline Slightly too underweight & 944 & 48 & $5 \cdot 1$ & 836 & $88 \cdot 6$ & 60 & $6 \cdot 4$ \\
\hline Proper weight & 4204 & 234 & $5 \cdot 6$ & 3812 & $90 \cdot 7$ & 158 & $3 \cdot 8$ \\
\hline Slightly too overweight & 679 & 100 & $14 \cdot 7$ & 564 & $83 \cdot 1$ & 15 & $2 \cdot 2$ \\
\hline Much too overweight & 58 & 14 & $24 \cdot 1$ & 41 & $70 \cdot 7$ & 3 & $5 \cdot 2$ \\
\hline
\end{tabular}

UdR, under-report; PR, plausible report; OvR, over-report.

*Weight categories according to International Obesity Taskforce criteria ${ }^{(18,19)}$.

Table 3 Descriptive analyses of continuous covariables stratified by reporting group (means and standard deviations): children aged 2-9 years, IDEFICS Study

\begin{tabular}{|c|c|c|c|c|c|c|c|c|}
\hline & \multicolumn{2}{|c|}{$\begin{array}{l}\text { Total group } \\
(n \text { 5962) }\end{array}$} & \multicolumn{2}{|c|}{$\begin{array}{c}\text { UdR } \\
(\text { n 402) }\end{array}$} & \multicolumn{2}{|c|}{$\begin{array}{c}\mathrm{PR} \\
(n \text { 5319) }\end{array}$} & \multicolumn{2}{|c|}{$\begin{array}{c}\text { OvR } \\
(n 241)\end{array}$} \\
\hline & Mean & SD & Mean & SD & Mean & SD & Mean & SD \\
\hline Age (years) & $6 \cdot 06$ & $1 \cdot 82$ & $6 \cdot 64$ & $1 \cdot 54$ & $6 \cdot 02$ & $1 \cdot 84$ & $5 \cdot 96$ & $1 \cdot 76$ \\
\hline BMl Z-score* & $0 \cdot 31$ & $1 \cdot 34$ & $0 \cdot 82$ & $1 \cdot 60$ & $0 \cdot 29$ & $1 \cdot 31$ & -0.01 & $1 \cdot 24$ \\
\hline El $(k J / d)$ & 6602 & 2218 & 3197 & 1021 & 6632 & 1807 & 11590 & 1833 \\
\hline $\mathrm{EI}(\mathrm{kcal} / \mathrm{d})$ & 1578 & 530 & 764 & 244 & 1585 & 432 & 2770 & 438 \\
\hline Water intake (g/d) & 319 & 357 & 284 & 346 & 317 & 352 & 419 & 462 \\
\hline$\%$ El from chocolate & $3 \cdot 2$ & 5.9 & $2 \cdot 7$ & 6.5 & $3 \cdot 2$ & 5.9 & 3.5 & $6 \cdot 7$ \\
\hline \%El from milk & $10 \cdot 1$ & $9 \cdot 4$ & $8 \cdot 5$ & $11 \cdot 3$ & $10 \cdot 3$ & $9 \cdot 2$ & $7 \cdot 9$ & $8 \cdot 7$ \\
\hline$\%$ El from soft drinks & $2 \cdot 7$ & $5 \cdot 7$ & $2 \cdot 7$ & 6.7 & $2 \cdot 7$ & $5 \cdot 7$ & $2 \cdot 2$ & $4 \cdot 0$ \\
\hline \%El from sugary products & $9 \cdot 8$ & $11 \cdot 7$ & $7 \cdot 4$ & $11 \cdot 8$ & $9 \cdot 8$ & $11 \cdot 6$ & $12 \cdot 7$ & $12 \cdot 4$ \\
\hline$\%$ El from fruits/vegetables & $8 \cdot 5$ & $8 \cdot 2$ & $11 \cdot 1$ & $13 \cdot 0$ & $8 \cdot 4$ & $7 \cdot 7$ & $6 \cdot 7$ & $6 \cdot 0$ \\
\hline
\end{tabular}

UdR, under-report; PR, plausible report; OvR, over-report; El, energy intake; \%El, percentage of energy intake.

${ }^{*}$ According to Cole et al. ${ }^{(31,32)}$. 
Table $4 \mathrm{OR}$ and $95 \% \mathrm{Cl}$ for the associations between overweight/obesity and El (Model 1a to $6 \mathrm{a}$ ), \%El from fruits/vegetables (Model $1 \mathrm{~b}$ to $6 \mathrm{~b}$ ) and \%El from soft drinks (Model $1 \mathrm{c}$ to $6 \mathrm{c}$ ) in different models: children aged 2-9 years, IDEFICS Study

\begin{tabular}{|c|c|c|c|c|c|c|c|c|c|c|c|c|}
\hline & \multicolumn{2}{|c|}{ Basic model } & \multicolumn{2}{|c|}{$\begin{array}{l}\text { Basic model adjusted } \\
\text { for covariables }\end{array}$} & \multicolumn{2}{|c|}{$\begin{array}{l}\text { Exclusion of } \\
\text { misreports }\end{array}$} & \multicolumn{2}{|c|}{$\begin{array}{l}\text { Adjustment for } \\
\text { reporting group }\end{array}$} & \multicolumn{2}{|c|}{$\begin{array}{l}\text { Adjustment for } \\
\text { propensity score }\end{array}$} & \multicolumn{2}{|c|}{$\begin{array}{l}\text { Adjustment for reporting group } \\
\text { and propensity score }\end{array}$} \\
\hline & OR & $95 \% \mathrm{Cl}$ & OR & $95 \% \mathrm{Cl}$ & OR & $95 \% \mathrm{Cl}$ & OR & $95 \% \mathrm{Cl}$ & OR & $95 \% \mathrm{Cl}$ & OR & $95 \% \mathrm{Cl}$ \\
\hline & \multicolumn{2}{|c|}{ Model $1 a^{*}$} & \multicolumn{2}{|c|}{ Model 2at } & \multicolumn{2}{|c|}{ Model 3ał } & \multicolumn{2}{|c|}{ Model 4a§ } & \multicolumn{2}{|c|}{ Model 5all } & \multicolumn{2}{|c|}{ Model 6a } \\
\hline $\begin{array}{l}\text { El }(1 \text { unit }=418 \cdot 4 \mathrm{~kJ} \\
(100 \mathrm{kcal})) \\
\text { PR } v . \text { UdR } \\
\text { OvR } v . \text { UdR } \\
\text { Propensity score }\end{array}$ & 0.996 & $0.983,1 \cdot 010$ & $1 \cdot 013$ & $0.995,1.031$ & 1.057 & $1 \cdot 038,1 \cdot 076$ & $\begin{array}{l}1 \cdot 068 \\
0 \cdot 205 \\
0 \cdot 041\end{array}$ & $\begin{array}{l}1 \cdot 049,1 \cdot 086 \\
0 \cdot 155,0.271 \\
0.023,0.073\end{array}$ & 1.019 & $1 \cdot 202,1 \cdot 243$ & $\begin{array}{l}0 \cdot 390 \\
0 \cdot 076 \\
1 \cdot 217\end{array}$ & $\begin{array}{l}1.054,1 \cdot 095 \\
0 \cdot 280,0.542 \\
0.041,0 \cdot 142 \\
1.038,1 \cdot 402\end{array}$ \\
\hline Propensity score & \multicolumn{2}{|c|}{ Model $1 b^{\star}$} & \multicolumn{2}{|c|}{ Model 2bt } & \multicolumn{2}{|c|}{ Model 3b $\ddagger$} & \multicolumn{2}{|c|}{ Model 4b§ } & \multicolumn{2}{|c|}{ Model 5b\| } & \multicolumn{2}{|c|}{ Model 6b- } \\
\hline $\begin{array}{l}\text { \%El from fruits/vegetables } \\
\text { PR } v . \text { UdR } \\
\text { OvR } v . \text { UdR } \\
\text { Propensity score }\end{array}$ & 1.009 & $1 \cdot 001,1 \cdot 018$ & 1.009 & $0.998,1.020$ & $\overline{1.007}$ & $0.998,1 \cdot 017$ & $\begin{array}{l}1.006 \\
0.365 \\
0.154\end{array}$ & $\begin{array}{l}0.997,1.014 \\
0.289,0.461 \\
0.099,0.242\end{array}$ & $1 \cdot 250$ & $1 \cdot 227,1 \cdot 274$ & $\begin{array}{l}0 \cdot 993 \\
0 \cdot 710 \\
0 \cdot 298 \\
1 \cdot 245\end{array}$ & $\begin{array}{l}0.984,1 \cdot 002 \\
0.532,0.948 \\
0 \cdot 181,0.491 \\
1.222,1.269\end{array}$ \\
\hline & \multicolumn{2}{|c|}{ Model $1 c^{*}$} & \multicolumn{2}{|c|}{ Model 2ct } & \multicolumn{2}{|c|}{ Model 3c $\ddagger$} & \multicolumn{2}{|c|}{ Model $4 c \S$} & \multicolumn{2}{|c|}{ Model 5c\| } & \multicolumn{2}{|c|}{ Model 6c- } \\
\hline $\begin{array}{l}\text { \%El from soft drinks } \\
\text { PR } v \text {. UdR } \\
\text { OvR } v . \text { UdR } \\
\text { Propensity score }\end{array}$ & 0.999 & $0.986,1 \cdot 013$ & 0.996 & $0.982,1.011$ & 0.996 & $0.982,1.011$ & $\begin{array}{l}1.001 \\
0.359 \\
0.151\end{array}$ & $\begin{array}{l}0.988,1.015 \\
0.285,0.453 \\
0.097,0.237\end{array}$ & 1.016 & $1 \cdot 000,1 \cdot 031$ & $\begin{array}{l}1 \cdot 015 \\
0 \cdot 692 \\
0 \cdot 307 \\
1 \cdot 226\end{array}$ & $\begin{array}{l}1 \cdot 000,1 \cdot 031 \\
0 \cdot 520,0 \cdot 921 \\
0 \cdot 188,0 \cdot 504 \\
1 \cdot 205,1 \cdot 248\end{array}$ \\
\hline
\end{tabular}

EI, energy intake; \%El, percentage of energy intake; PR, plausible report; UdR, under-report; OvR, over-report.

Effects of continuous variables are assessed as 1 -unit offsets from the mean. Due to the small scale of the propensity score, 0.01 -unit offsets from mean were chosen here.

'Basic model. logistic multilevel regression model; OR for the association between overweight/obesity and food intake adjusted for age and sex and including the study centre as random effect ( $n 5962$ ).

+Basic model additionally adjusted for net household income (dummy: high $v$. medium/low), number of persons below 18 years of age in the household, day of the interview (dummy: weekday $v$. Saturday/Sunday),

information on parental concerns and perception regarding their child's weight status and reported intakes from food groups associated with misreporting.

\#Basic model, but excluding UdR and OvR ( $n$ 5319).

Basic model adjusted for the reporting group (UdR, PR, OvR).

Basic model adjusted for a propensity score for misreporting.

'Basic model adjusted for the reporting group and for the propensity score for misreporting. 
between overweight/obesity and fruit/vegetable intake being reversed compared with the basic model. Significant associations were found between overweight/obesity and EI as well as soft drink intake. Finally, adjustment for the reporting group and propensity score at the same time strengthened the association between overweight/obesity and EI whereas the other associations remained nearly unchanged (Models 6a-c) compared with the model adjusting only for the propensity score.

When stratifying the basic model by the reporting group (Table 5, Model $7 \mathrm{a}-\mathrm{c}$ ), only EI was significantly related to overweight/obesity in all three strata. Additional adjustment for the propensity score (Model $8 \mathrm{a}-\mathrm{c}$ ) strengthened associations between all three dietary exposures and overweight/obesity. Here a significant reverse association between fruit/vegetable intake and overweight/obesity was observed in OvR and a positive association was found between soft drinks and overweight/obesity in UdR. The relationship between overweight/obesity and EI was much stronger in the UdR and OvR groups compared with PR.

\section{Discussion}

To the authors' knowledge, the present study is the first one in children applying and comparing several statistical approaches to counteract attenuation of risk estimates caused by misreporting of dietary information. Negligence of misreporting in the statistical model revealed insignificant or even (unexpected) reversed diet-obesity associations. Consistent with previous findings on differential misreporting by weight status ${ }^{(33)}$, the UdR group had higher mean BMI Z-scores but reported lower (implausible) EI compared with PR. The opposite was true for the OvR group. Such reporting bias may obscure positive relationships between diet and weight status. Researchers should be aware that results may differ strongly depending on the statistical model selected and that the choice of an adequate model needs to be taken thoroughly. Consideration of misreporting in any way yielded results more consistent with hypotheses relating food intake to overweight/obesity ${ }^{(34,35)}$. However, the true effects remained unknown due to the lack of validation data. A recent study reported that not excluding implausible reports resulted in weak, nonsignificant or even misleading associations between BMI and $\operatorname{diet}^{(9)}$, whereas Nielsen and Adair stated that examining all data but stratifying by level of intake may be more informative for population nutrient intake than exclusion of misreports ${ }^{(8)}$. Savage et al. found a significant association between BMI and reported EI in the PR of preadolescent girls, but neither in the total study group nor when analysing only misreports (combining UdR and OvR into one group) ${ }^{(36)}$. This agrees with our results for the total study group (basic model). Nevertheless, our stratified analysis revealed statistically significant associations between overweight/obesity and EI in all three reporting 
groups, being even stronger in UdR and OvR compared with PR. This may be explained by either: (i) differences in the mean intake levels to which the effects are put into relation (mean EI: $3197 \mathrm{~kJ} / \mathrm{d}(764 \mathrm{kcal} / \mathrm{d})$ in UdR, $6632 \mathrm{~kJ} / \mathrm{d}$ (1585 kcal/d) in PR, $11590 \mathrm{~kJ} / \mathrm{d}(2770 \mathrm{kcal} / \mathrm{d})$ in OvR); or (ii) differences between the reporting groups in terms of participants' characteristics (e.g. prevalence of overweight/ obesity: $38.0 \%$ in UdR, $19 \cdot 9 \%$ in PR, $13.2 \%$ in OvR). Our results argue against combining UdR and OvR into one group in stratified analyses as determinants of misreporting and participants' characteristics are likely to differ ${ }^{(30)}$. Moreover, the differences between the groups of UdR, PR and OvR suggest that data exclusions may actually introduce a selection bias, so that exclusion of misreports is not recommended. However, the reduced sample sizes resulting from both data exclusions and stratification go along with limited statistical power especially in the (smaller) groups of UdR and OvR. Adjustment for the reporting group does not affect the statistical power to such a degree and shifted associations between overweight/ obesity and all three dietary exposures to the expected directions (Models $4 \mathrm{a}-\mathrm{c}$ ). These results agree with those from a study by Mendez et al. ${ }^{(10)}$ where associations between different food groups and overweight/obesity became stronger after inclusion of dummy variables identifying under- and over-reports. In that study, dummy adjustment revealed results similar to those obtained when limiting the analysis sample to plausible reports, as observed in our study. However, this approach has the disadvantage of misclassifications of single recalls being quite likely, which may again bias the results ${ }^{(37)}$.

After adjustment for the propensity score, which combined various indicators for misreporting into one summary measure, associations between overweight/ obesity and soft drink as well as fruit/vegetable intakes increased markedly. To correct for selective reporting of single food items, also dietary variables commonly associated with misreporting were included when constructing the propensity score. This approach strived for an effect similar to regression calibration ${ }^{(38)}$ although both procedures differ. The idea of calibration in general is the replacement of exposures measured with error by 'adjusted' values using additional information obtained from biomarker measurements or from a second dietary assessment instrument. Common calibration approaches assume (non-differential) linear measurement error with constant variance or linear random within-person error in the case of replicate measurements (e.g. repeated $24-\mathrm{HDR})^{(38-40)}$ - assumptions that are often violated due to differential misreporting ${ }^{(4,41)}$. Moreover, error structures were found to be correlated when assessing dietary information via different assessment methods (e.g. FFQ and 24-HDR) ${ }^{(42)}$. Although the use of two complementary dietary assessment methods is recommended e.g. when investigating usual intakes ${ }^{(43,44)}$, the benefit of a second assessment instrument to correct for misreporting is questionable ${ }^{(45)}$. Further studies are needed to explore and compare the calibration and propensity score approach. However, it can be suspected that statistical adjustment of relative risks based on biomarker data with independent error structures (e.g. doubly labelled water for EI) incorporating characteristics of misreporters should be preferred if such data exist ${ }^{(1,39,46)}$. In the absence of validation data, the propensity score seems to be a useful, cost-effective alternative to account for misreporting.

In our models, intakes from soft drinks and fruits/ vegetables were examined in relation to total daily intake of energy (expressed as \%EI) instead of including absolute amounts $(\mathrm{g} / \mathrm{d})$. Use of absolute amounts would result in lower effects in high energy consumers compared with low energy consumers ${ }^{(3,47)}$. To overcome this problem, different energy adjustment models have been proposed next to the one applied here ${ }^{(48)}$. But again energy adjustment cannot eliminate differential biases ${ }^{(3)}$ and is therefore not sufficient to correct for subject-specific and selective misreporting of certain foods/macronutrients ${ }^{(45,49)}$. The advantage of additional incorporation of the propensity score over simple energy-adjustment methods is that the propensity score is a comprehensive approach to account for several covariables related to misreporting instead of considering only the level of EI. Under-reporting is difficult to distinguish from undereating (defined as eating less than required to maintain body weight, accompanied by weight loss) but both are treated equally in energy-adjustment models, while it can be hypothesised that subject characteristics and therefore propensity scores differ between undereaters and under-reporters. Nevertheless, in the case of non-differential errors energyadjustment methods were shown to be a good approach to counteract underestimation of relative risks and reduction of statistical power ${ }^{(1)}$.

Several sensitivity analyses were carried out (e.g. including only children with two repeated 24-HDR ( $n$ 904), excluding OvR ( $n$ 241), excluding UdR ( $n$ 402), excluding $24-H D R$ with at least one imputed value ( $n$ 69), excluding thin ( $n$ 556) or obese children $(n$ 430)). When including only children with two repeated $24-\mathrm{HDR}$, model estimates became unstable due to the reduction in sample size. In all other cases, results remained nearly unchanged compared with the results given here. Details can be obtained from the author on request.

The present analysis is based on data in children relying on proxy reports. Here misreporting may result not only from intentional misreporting, e.g. caused by social desirability or parental concerns about their child's weight status, but also from unintentional misreporting due to lack of parental control (out-of-home meals). Our discussion mainly refers to studies in adolescents/adults as related studies are lacking in children. Although determinants for misreporting may differ between children and adolescent/ adult populations, previous studies and the present one reveal similar results concerning the statistical approaches 
of data exclusions, stratification or adjustment for the reporting group. Nevertheless, results of the newly applied propensity score approach should not simply be transferred. When applying the propensity score approach in future studies, variables for the construction of the score should be selected depending on the study population under investigation, which may require a pre-study to identify the relevant determinants of misreporting. The analysis of the usefulness of the propensity score adjustment in adolescent/adult populations is a task for future research.

\section{Limitations and strengths}

Only one recall day per child was used in the present analysis which does not reflect usual intakes due to the dayto-day variation that characterises dietary data in general ${ }^{(50)}$. Day-to-day variation results in random (non-differential) errors that may have weakened associations between dietary factors and overweight/obesity. In addition, extreme intakes may not necessarily reflect misreporting but rather specific diets (e.g. energy restricted) or exceptional days (e.g. the child was ill or extremely physically active). Reverse causation cannot be precluded as obesity may even cause low intakes due to dieting or change in eating behaviour. Causal inference is limited owing to the crosssectional study design.

Sensitivity of the cut-off technique to correctly classify UdR and OvR is limited as it aims only to identify misreports resulting in physiologically implausibly low/high $\mathrm{EI}^{(6)}$. By application of the cut-off technique distinction between varying degrees of misreporting is not feasible; e.g. under-reporting from a high intake level may not be detected as the reported intake may still be such high that EI:BMR does not fall below the cut-off. Furthermore, not considering individual physical activity levels of the children when classifying the 24-HDR is a limitation. Physically inactive children may have a very low daily energy expenditure making even low reported intakes plausible, whereas physically active children have an increased likelihood to be misclassified as OvR. Childspecific reference PAL were used in the calculation of the cut-offs to compensate for the lack of sufficient individual information on physical activity.

The study was a first exploratory approach to investigate the usefulness of propensity scores in the context of dietary misreporting in children. The authors are aware that there are several different ways to construct a propensity score by inclusion of additional/different variables, e.g. physical activity, number of daily meals, etc. The rather exploratory character of the paper should be underlined here. However, the application of the new propensity score approach, along with the large sample size, the variety of covariables and the standardised assessment procedures suggest that the present study provides important knowledge on methods to handle misreporting in future research, while also highlighting gaps in knowledge as starting points for further analyses.

\section{Conclusions}

Associations between dietary exposures and health outcomes are strongly affected or even masked or reversed by measurement errors. Instead of data exclusions that may result in unknown bias, misreporting should rather be addressed in the model building process including adjustment terms for misreporting. Dummy adjustment for the reporting group revealed associations more consistent with expectations, which was most pronounced considering the association between EI and overweight/obesity. However, more sophisticated adjustments seem to be necessary to counteract the effect of selective misreporting of other food groups. In this respect, the propensity score adjustment turned out to be a useful tool to correct for subject-specific misreporting as it combines various variables associated with misreporting into one scalar and should be further investigated in future studies.

\section{Acknowledgements}

Sources of funding: This work was done as part of the IDEFICS Study (www.idefics.eu) and is published on behalf of its European Consortium. Financial support was provided by the European Community within the Sixth RTD Framework Programme Contract No. 016181 (FOOD). S.B.-S. was funded by a grant from the Aragón's Regional Government (Diputación General de Aragón, DGA). B.V. was funded by the Research FoundationFlanders (FWO). Conflict of interest: All the authors declare that there are no conflicts of interest. Authors' contributions: All authors contributed to conception and design, acquisition of data, analysis or interpretation of data. Each author has seen and approved the contents of the submitted manuscript. Final approval of the version published was given by all authors.

\section{References}

1. Freedman LS, Schatzkin A, Midthune D et al. (2011) Dealing with dietary measurement error in nutritional cohort studies. J Natl Cancer Inst 103, 1086-1092.

2. Lioret S, Touvier M, Balin M et al. (2011) Characteristics of energy under-reporting in children and adolescents. Br J Nutr 105, 1671-1680.

3. Livingston MB \& Black AE (2003) Markers of the validity of reported energy intake. J Nutr 133, Suppl. 3, 895S-920S.

4. Black AE \& Cole TJ (2001) Biased over- or under-reporting is characteristic of individuals whether over time or by different assessment methods. J Am Diet Assoc 101, 70-80.

5. Shai I, Rosner BA, Shahar DR et al. (2005) Dietary evaluation and attenuation of relative risk: multiple comparisons between blood and urinary biomarkers, food frequency, and 24-hour recall questionnaires: the DEARR study. J Nutr 135, 573-579.

6. Goldberg GR, Black AE, Jebb SA et al. (1991) Critical evaluation of energy intake data using fundamental principles of energy physiology: 1 . Derivation of cut-off limits to identify under-recording. Eur J Clin Nutr 45, 569-581. 
7. McCrory MA, Hajduk CL \& Roberts SB (2002) Procedures for screening out inaccurate reports of dietary energy intake. Public Health Nutr 5, 873-882.

8. Nielsen SJ \& Adair L (2007) An alternative to dietary data exclusions. J Am Diet Assoc 107, 792-799.

9. Huang TT, Roberts SB, Howarth NC et al. (2005) Effect of screening out implausible energy intake reports on relationships between diet and BMI. Obes Res 13, 1205-1217.

10. Mendez MA, Wynter S, Wilks R et al. (2004) Under- and overreporting of energy is related to obesity, lifestyle factors and food group intakes in Jamaican adults. Public Health Nutr 7, 9-19.

11. Howarth NC, Huang TT, Roberts SB et al. (2005) Dietary fiber and fat are associated with excess weight in young and middle-aged US adults. J Am Diet Assoc 105 , $1365-1372$

12. Gibson RS (2005) Principles of Nutritional Assessment, 2nd ed. New York: Oxford University Press.

13. Rosenbaum PR \& Rubin DB (1983) The central role of the propensity score in observational studies for causal effects. Biometrika 70, 41-55.

14. Mendez MA, Popkin BM, Buckland G et al. (2011) Alternative methods of accounting for underreporting and overreporting when measuring dietary intake-obesity relations. Am J Epidemiol 173, 448-458.

15. Livingstone MB \& Robson PJ (2000) Measurement of dietary intake in children. Proc Nutr Soc 59, 279-293.

16. Ahrens W, Bammann K, de Henauw S et al. (2006) Understanding and preventing childhood obesity and related disorders - IDEFICS: a European multilevel epidemiological approach. Nutr Metab Cardiovasc Dis 16, 302-308.

17. Ahrens W, Bammann K, Siani A et al. (2011) The IDEFICS cohort: design, characteristics and participation in the baseline survey. Int J Obes (Lond) 35, Suppl 1, S3-S15.

18. Cole TJ, Bellizzi MC, Flegal KM et al. (2000) Establishing a standard definition for child overweight and obesity worldwide: international survey. BMJ 320, 1240-1243.

19. Cole TJ, Flegal KM, Nicholls D et al. (2007) Body mass index cut offs to define thinness in children and adolescents: international survey. BMJ 335, 194.

20. Vereecken CA, Covents M, Sichert-Hellert W et al. (2008) Development and evaluation of a self-administered computerized 24-h dietary recall method for adolescents in Europe. Int J Obes (Lond) 32, Suppl. 5, S26-S34.

21. Vereecken CA, Covents M, Matthys C et al. (2005) Young adolescents' nutrition assessment on computer (YANA-C). Eur J Clin Nutr 59, 658-667.

22. Zurriaga O, Perez-Panades J, Quiles Izquierdo J et al (2011) Factors associated with childhood obesity in Spain. The OBICE study: a case-control study based on sentinel networks. Public Health Nutr 14, 1105-1113.

23. O'Connor TM, Yang SJ \& Nicklas TA (2006) Beverage intake among preschool children and its effect on weight status. Pediatrics 118, e1010-e1018.

24. Alinia S, Hels O \& Tetens I (2009) The potential association between fruit intake and body weight - a review. Obes Rev 10, 639-647.

25. Schofield WN (1985) Predicting basal metabolic rate, new standards and review of previous work. Hum Nutr Clin Nutr 39, Suppl. 1, 5-41.

26. Sichert-Hellert W, Kersting M \& Schoch G (1998) Underreporting of energy intake in 1 to 18 year old German children and adolescents. Z Ernabrungswiss 37, 242-251.

27. Nelson M, Black AE, Morris JA et al. (1989) Between- and within-subject variation in nutrient intake from infancy to old age: estimating the number of days required to rank dietary intakes with desired precision. Am J Clin Nutr 50, $155-167$.
28. Black AE (2000) Critical evaluation of energy intake using the Goldberg cut-off for energy intake:basal metabolic rate. A practical guide to its calculation, use and limitations. Int J Obes Relat Metab Disord 24, 1119-1130.

29. Torun B, Davies PS, Livingstone MB et al. (1996) Energy requirements and dietary energy recommendations for children and adolescents 1 to 18 years old. Eur J Clin Nutr 50, Suppl.1, S37-S80.

30. Börnhorst C, Huybrechts I, Ahrens W et al. (2012) Prevalence and determinants of misreporting among European children in proxy-reported 24-hour dietary recalls. $\mathrm{Br} \quad \mathrm{I}$ Nutr (Epublication ahead of print version).

31. Cole TJ, Freeman JV \& Preece MA (1998) British 1990 growth reference centiles for weight, height, body mass index and head circumference fitted by maximum penalized likelihood. Stat Med 17, 407-429.

32. Cole TJ, Freeman JV \& Preece MA (1995) Body mass index reference curves for the UK, 1990. Arch Dis Child 73, 25-29.

33. Heitmann BL, Lissner L \& Osler M (2000) Do we eat less fat, or just report so? Int J Obes Relat Metab Disord 24, 435-442.

34. Tohill BC, Seymour J, Serdula M et al. (2004) What epidemiologic studies tell us about the relationship between fruit and vegetable consumption and body weight. Nutr Rev 62 , 365-374.

35. Rolls BJ, Drewnowski A \& Ledikwe JH (2005) Changing the energy density of the diet as a strategy for weight management. J Am Diet Assoc 105, 5 Suppl. 1, S98-S103.

36. Savage JS, Mitchell DC, Smiciklas-Wright H et al. (2008) Plausible reports of energy intake may predict body mass index in pre-adolescent girls. J Am Diet Assoc 108, $131-135$

37. Greenland S \& Robins JM (1985) Confounding and misclassification. Am J Epidemiol 122, 495-506.

38. Spiegelman D, McDermott A \& Rosner B (1997) Regression calibration method for correcting measurement-error bias in nutritional epidemiology. Am J Clin Nutr 65, 4 Suppl., 1179S-1186S.

39. Freedman LS, Midthune D, Carroll RJ et al. (2011) Using regression calibration equations that combine self-reported intake and biomarker measures to obtain unbiased estimates and more powerful tests of dietary associations. Am J Epidemiol 174, 1238-1245.

40. Kaaks R, Riboli E \& van Staveren W (1995) Calibration of dietary intake measurements in prospective cohort studies. Am J Epidemiol 142, 548-556.

41. Livingstone MB, Robson PJ, Black AE et al. (2003) An evaluation of the sensitivity and specificity of energy expenditure measured by heart rate and the Goldberg cut-off for energy intake: basal metabolic rate for identifying mis-reporting of energy intake by adults and children: a retrospective analysis. Eur J Clin Nutr 57, 455-463.

42. Kipnis V, Subar AF, Midthune D et al. (2003) Structure of dietary measurement error: results of the OPEN biomarker study. Am J Epidemiol 158, 14-21.

43. Carroll RJ, Midthune D, Subar AF et al. (2012) Taking advantage of the strengths of 2 different dietary assessment instruments to improve intake estimates for nutritional epidemiology. Am J Epidemiol 175, 340-347.

44. de Boer EJ, Slimani N, van't Veer P et al. (2011) The European Food Consumption Validation Project: conclusions and recommendations. Eur J Clin Nutr 65, Suppl.1, S102-S107.

45. Westerterp KR \& Goris AH (2002) Validity of the assessment of dietary intake: problems of misreporting. Curr Opin Clin Nutr Metab Care 5, 489-493.

46. Freedman LS, Tasevska N, Kipnis V et al. (2010) Gains in statistical power from using a dietary biomarker in combination with self-reported intake to strengthen the 
analysis of a diet-disease association: an example from CAREDS. Am J Epidemiol 172, 836-842.

47. Willett W \& Stampfer MJ (1986) Total energy intake: implications for epidemiologic analyses. Am J Epidemiol 124, 17-27.

48. Kipnis V, Freedman LS, Brown CC et al. (1997) Effect of measurement error on energy-adjustment models in nutritional epidemiology. Am J Epidemiol 146, 842-855.
49. Lafay L, Mennen L, Basdevant A et al. (2000) Does energy intake underreporting involve all kinds of food or only specific food items? Results from the Fleurbaix Laventie Ville Sante (FLVS) study. Int J Obes Relat Metab Disord 24, 1500-1506.

50. Nielsen SB, Montgomery C, Kelly LA et al. (2008) Energy intake variability in free-living young children. Arch Dis Child 93, 971-973. 